\title{
CRECIMIENTO MiCELIAR DE ESPECIES SILVESTRES DE HONGOS COMESTIBLES DE LOS BOSQUES ANDINO-PATAGÓNICOS: PRIMEROS PASOS PARA SU DOMESTICACIÓN
}

\author{
CAROLINA VERÓNICA TOLEDO ${ }^{1}$ y CAROLINA BARROETAVEÑA ${ }^{1,2,3, *}$
}

\begin{abstract}
Summary: Myceliar growth of wild species of edible fungi of the Andean-Patagonian forests: first steps for their domestication. Nothofagus spp. forests from Patagonia Argentina host fungi with organoleptic and nutritional characteristics that make them attractive for consumption and plausible to be cultivated on different organic substrates. The optimum parameters of vegetative growth of seven potentially cultivable species of edible wild mushrooms in these forests (Aleurodiscus vitellinus, Fistulina antarctica, F. endoxantha, Grifola gargal, Lepista nuda, Lycoperdon perlatum and Macrolepiota afín procera) were evaluated. Five strains of each of the fungal species were obtained, and three assays were performed: 1) determining the optimum temperature for mycelia growth, 2) assessment of miceliar growth on different culture media and characterization of the strains, and 3) assessment of myceliar growth in grains. The optimum growth temperature was generally $25^{\circ} \mathrm{C}$, although with varying ranges. The culture media more rapidly colonized was Potato Dextrose Agar. Lepista nuda and $A$. vitellinus showed the highest rates of average myceliar growth both in culture media and in grains. Strains of $A$. vitellinus, $F$. endoxantha and $L$. perlatum showed greater heterogeneity in the rate of growth in plates, while strains of $F$. antarctica, $G$. gargal and $M$. afín procera showed a more homogeneous behavior.
\end{abstract}

Keys words: Primary inoculum, domestication, growth temperature, growth in different media.

Resumen: Los bosques de Nothofagus spp. de la Patagonia Argentina albergan hongos con características organolépticas y nutricionales que las tornan atractivas para su consumo y plausibles de ser cultivadas sobre diferentes sustratos orgánicos. Se evaluaron los parámetros óptimos de crecimiento vegetativo de 7 especies de hongos silvestres comestibles potencialmente cultivables que pueden ser encontrados en estos bosques (Aleurodiscus vitellinus, Fistulina antarctica, F. endoxantha, Grifola gargal, Lepista nuda, Lycoperdon perlatum y Macrolepiota afín procera). Se obtuvieron 5 cepas de cada especie y se realizaron tres ensayos: 1) determinación de la temperatura óptima de crecimiento, 2) evaluación del crecimiento en diferentes medios de cultivo y caracterización miceliar de las cepas, y 3) evaluación del crecimiento de los micelios en granos. La temperatura óptima de crecimiento fue en general de 25 ${ }^{\circ} \mathrm{C}$, aunque con rangos variables. El medio de cultivo más rápidamente colonizado fue el agar con papa y dextrosa. Lepista nuda y A. vitellinus mostraron las mayores tasas de crecimiento miceliar promedio tanto en medios como en granos. Las cepas de A. vitellinus, $F$. endoxantha y L. perlatum mostraron mayor heterogeneidad en la tasa de crecimiento en placas, mientras que las cepas de F. antarctica, G. gargal y $M$. afín procera mostraron comportamientos homogéneos.

Palabras clave: Inóculo primario, domesticación, temperatura de crecimiento, crecimiento en diferentes medios.

${ }^{1}$ Centro de Investigación y Extensión Forestal Andino Patagónico (CIEFAP). Ruta 259 Km 4 Esquel (9200), Chubut, Argentina.

${ }^{2}$ Universidad Nacional de la Patagonia San Juan Bosco, Facultad de Ingeniería. Ruta 259 Km 4 Esquel (9200), Chubut, Argentina.

${ }^{3}$ Consejo Nacional de Investigaciones Científicas y Tecnológicas (CONICET), Argentina.

* Correo electrónico: cbarroetaveña@correociefap.org.ar 


\section{INTRODUCCIÓN}

El cultivo de hongos comestibles es una actividad productiva que se ha desarrollado ampliamente en diversas partes del mundo, especialmente en Europa y Asia (Wang, 1987; Boa, 2004). Las especies más cultivadas mundialmente son Agaricus bisporus (J.E. Lange) Imbach, Lentinula edodes (Berk.) Pegler y Pleurotus spp., que representan aproximadamente el $75 \%$ del volumen que se comercializa (Chang \& Miles, 2004). En los últimos años la demanda de hongos comestibles en el mercado de alimentos se ha incrementado (Boa, 2004), dado que constituyen alimentos saludables, bajos en calorías y ricos en proteínas, vitaminas, hierro, zinc, selenio, sodio, quitina, fibras y minerales (Chang \& Miles, 2004).

Los bosques nativos de la región andinopatagónica de Argentina, mayormente dominados por Nothofagus spp., albergan numerosas especies de hongos, algunas de las cuales han sido reportadas como comestibles y poseen características organolépticas, tales como aroma, sabor, textura y color, junto a tamaño y formas interesantes o novedosas, que las tornan atractivas y potencialmente aptas para su cultivo con fines comerciales (Gamundí \& Horak, 1993; Valenzuela-Flores, 2003; Furci, 2007; Gorjón, et al., 2013; Toledo et al. 2014; 2016). En particular las especies lignícolas Aleurodiscus vitellinus (Lév.) Pat., Fistulina antarctica Speg., $F$. endoxantha Speg. y Grifola gargal Singer, junto a las especies humícolas Macrolepiota afín procera (Scop.) Singer, Lepista nuda (Bull.) Cooke y Lycoperdon perlatum Pers., todas capaces de degradar el sustrato orgánico sobre el que se desarrollan, se presentan como atractivas y plausibles de cultivar de manera intensiva sobre diversos desechos agrícolas y forestales que se generan en el país. De todas ellas, existe actualmente registro de aprovechamiento silvestre de los pobladores criollos y mapuches de la región solo de G. gargal, F. antarctica y $F$. endoxantha (Toledo, 2016). Existen reportes de estudios relacionados con la domesticación solo para G. gargal, L. nuda y $M$. afín procera (Guinberteau \& Olivier, 1991; Stamets, 1993; Shim, et al., 2005; Gaitán-Hernández \& Báez Rodríguez, 2008; FIA, 2009; Castro, et al., 2014; Harada, et al., 2015).
Las ventajas asociadas al cultivo de especies de hongos silvestres se relacionan a su estacionalidad (producen basidiomas en un estrecho lapso durante el otoño y, eventualmente, en primaveras lluviosas) a las dificultades de recolección (los ambientes donde se desarrollan presentan mayormente accesibilidad reducida), y a la baja frecuencia de hallazgo comparada con especies asociadas a plantaciones, como Suillus luteus (L.) Roussel (Toledo, et al., 2014).

El cultivo intensivo de nuevas especies de hongos comestibles requiere de la obtención de cepas, de la determinación de la temperatura óptima de crecimiento en cultivo, de la velocidad de crecimiento vegetativo y de la calidad del micelio, como parámetros iniciales y necesarios (Gaitán-Hernández \& Báez Rodríguez, 2008; Bran et al., 2009; Suárez \& Holguín, 2011; LeónAvendaño et al., 2013). Seguidamente, es necesario seleccionar el grano más adecuado para producir inóculo primario en cada caso. El mejor medio o grano será aquel que es colonizado en menor tiempo, favoreciendo el acortamiento de los ciclos de producción de basidiomas y la reducción de incidencia de contaminantes (Stamets, 1993; Albertó, 2008).

Este trabajo abordó los pasos iniciales de la domesticación de las 7 especies de hongos humícolas/degradadoras citadas, específicamente evaluando: a) el crecimiento miceliar de las diferentes especies y cepas en placas de Petri a diferentes valores de temperatura $\left(20,25\right.$ y $\left.30^{\circ} \mathrm{C}\right)$ y usando tres medios de cultivo (AEM, APD y AM); b) las características morfológicas de los micelios en cada medio para cada una de las cepas, como un indicador de vigor, c) el crecimiento miceliar de las diferentes especies y cepas sobre sustratos a base de granos de trigo y centeno.

\section{Materiales y Métodos}

\section{Material biológico y medios de cultivo}

Se obtuvieron 5 cepas de cada una de las 7 especies de hongos comestibles seleccionadas (especies humícolas: L. nuda, L. perlatum y M. afín procera, degradadoras de la madera: A. vitellinus, $F$. antarctica, F. endoxantha, y G. gargal). Cada cultivo se aisló de diferentes basidiomas frescos en medio agar con extracto de malta (AEM). Los 


\section{V. Toledo y C. Barroetaveña - Caracterización miceliar de hongos silvestres comestibles}

especímenes fueron coleccionados en bosques de Nothofagus spp. en las provincias de Chubut, Río Negro y Neuquén, Argentina, determinados siguiendo a Moser (1978), Phillips (1981), Arora (1986), Gorjón et al. (2013) y Rajchenberg (2006) y depósitados en el Herbario del Centro de Investigación y Extensión Forestal Andino Patagónico (Tabla 1). Las cepas se mantuvieron en viales con agua destilada en la Colección de cultivos de hongos BAFCcult. (Cepario de la Facultad de Ciencias Exactas y NaturalesUniversidad de Buenos Aires, Argentina). Se utilizó como referencia para comparar velocidades de crecimiento con las cepas patagónicas, una cepa comercial (ICFC 32/99) de Pleurotus ostreatus (Jacq.) P. Kumm. (cepario IIB-INTECH, Argentina).

El crecimiento y características morfológicas de los micelios se evaluaron en tres medios de cultivo: agar con extracto de malta (AEM, Difco), agar de papa y dextrosa (APD, Difco) y agar con malta y maíz (AM, Difco). Se utilizaron placas de Petri plásticas de $80 \mathrm{~mm}$ x $12 \mathrm{~mm}$ conteniendo $20 \mathrm{ml}$ de medio, inoculadas en el centro con discos de 0,5 $\mathrm{cm}(\varnothing)$ de cultivos de cada cepa, crecidos en medio AEM durante 30 días.

\section{Determinación de la temperatura óptima de crecimiento miceliar}

Se inocularon 189 placas de Petri de $90 \mathrm{~mm}$ de diámetro con 3 cepas de cada especie (con un taco de micelio de $5 \mathrm{~mm}$ de diámetro) que fueron incubadas en oscuridad a 20,25 y $30{ }^{\circ} \mathrm{C}$ por 20 días en medio AEM. Se registró el diámetro de la colonia cada 48 $\mathrm{h}$ en dos ejes cartesianos trazados sobre las tapas de las cajas, y se calculó el promedio para cada par de datos. La tasa de crecimiento miceliar $(\mathrm{Kt})$ se estimó con la función de crecimiento lineal $y=k x+$ $c(y=$ distancia, $x=$ tiempo y $c=$ factor constante $)$ y se expresó en milímetros por día $(\mathrm{mm} / \mathrm{d})$ (Zervakis et al., 2001).

Evaluación del crecimiento y caracterización miceliar de las cepas en diferentes medios de cultivo

Luego de determinar la temperatura óptima de crecimiento, se inocularon 5 cepas de cada especie con un taco de micelio de $5 \mathrm{~mm}$ en placas de Petri de $90 \mathrm{~mm}$ de diámetro con AEM, APD, y AM, e incubaron en oscuridad a $25 \pm 1^{\circ} \mathrm{C}$ por 38 días.
Tabla 1. Listado de especies estudiadas con detalle del lugar de recolección, nombre del colector, $\mathrm{N}^{\circ}$ de colección y $\mathrm{N}^{\circ}$ BAFCcult.

\begin{tabular}{|c|c|c|c|}
\hline & $N^{\circ}$ BAFC & Procedencia & Sustrato \\
\hline \multirow{5}{*}{ 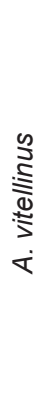 } & 4485 & $\begin{array}{c}7 \text { Lagos-PNNH- } \\
\text { Neuquén }\end{array}$ & $\begin{array}{l}\text { madera muerta } \\
\text { de } N \text {. dombeyi }\end{array}$ \\
\hline & 4483 & $\begin{array}{l}\text { Chachín-PNL- } \\
\text { Neuquén }\end{array}$ & $\begin{array}{l}\text { madera muerta } \\
\text { de } N \text {. dombeyi }\end{array}$ \\
\hline & 4490 & $\begin{array}{l}\text { Río Rivadavia- } \\
\text { PNLA-Chubut }\end{array}$ & $\begin{array}{l}\text { madera muerta } \\
\text { de } N \text {. dombeyi }\end{array}$ \\
\hline & 4467 & $\begin{array}{l}\text { Río Rivadavia- } \\
\text { PNLA-Chubut }\end{array}$ & $\begin{array}{l}\text { madera muerta } \\
\text { de } N \text {. dombeyi }\end{array}$ \\
\hline & 4484 & $\begin{array}{l}\text { Río Arrayanes- } \\
\text { PNLA-Chubut }\end{array}$ & $\begin{array}{l}\text { madera muerta } \\
\text { de } N \text {. dombeyi }\end{array}$ \\
\hline \multirow{5}{*}{ 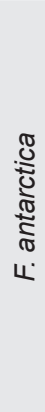 } & 4481 & $\begin{array}{l}\text { Reserva provincial } \\
\text { Bagillt-Chubut }\end{array}$ & $\begin{array}{l}\text { madera viva } \\
\text { de } N \text {. pumilio }\end{array}$ \\
\hline & 4474 & $\begin{array}{l}\text { Reserva provincial } \\
\text { Bagillt-Chubut }\end{array}$ & $\begin{array}{l}\text { madera viva } \\
\text { de } N \text {. pumilio }\end{array}$ \\
\hline & 4465 & $\begin{array}{l}\text { Río Arrayanes- } \\
\text { PNLA-Chubut }\end{array}$ & $\begin{array}{c}\text { madera viva } \\
\text { de } N \text {. dombeyi }\end{array}$ \\
\hline & 4479 & $\begin{array}{l}7 \text { Lagos-PNNH- } \\
\text { Neuquén }\end{array}$ & $\begin{array}{c}\text { madera viva } \\
\text { de } N \text {. dombeyi }\end{array}$ \\
\hline & 4482 & $\begin{array}{l}\text { Río Rivadavia- } \\
\text { PNLA-Chubut }\end{array}$ & $\begin{array}{c}\text { madera viva } \\
\text { de } N \text {. dombeyi }\end{array}$ \\
\hline \multirow{5}{*}{ 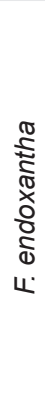 } & 4486 & $\begin{array}{l}\text { Chachín-PNL- } \\
\text { Neuquén }\end{array}$ & $\begin{array}{l}\text { madera viva } \\
\text { de } N \text {. obliqua }\end{array}$ \\
\hline & 4487 & $\begin{array}{l}\text { Chachín-PNL- } \\
\text { Neuquén }\end{array}$ & $\begin{array}{l}\text { madera viva } \\
\text { de } N \text {. obliqua }\end{array}$ \\
\hline & 4493 & $\begin{array}{l}\text { Ea Quechuquina- } \\
\text { PNL-Neuquén }\end{array}$ & $\begin{array}{l}\text { madera viva } \\
\text { de } N \text {. obliqua }\end{array}$ \\
\hline & 4476 & $\begin{array}{l}\text { Lago Queñi- } \\
\text { PNL-Neuquén }\end{array}$ & $\begin{array}{l}\text { madera viva } \\
\text { de } N \text {. obliqua }\end{array}$ \\
\hline & 4475 & $\begin{array}{l}\text { Pto. Pucará- } \\
\text { PNL-Neuquén }\end{array}$ & $\begin{array}{l}\text { madera viva } \\
\text { de } N \text {. alpina }\end{array}$ \\
\hline \multirow{5}{*}{$\begin{array}{l}\bar{\pi} \\
\frac{\$}{\pi} \\
\text { o } \\
\text { ن. }\end{array}$} & 4488 & $\begin{array}{l}\text { Pto. Pucará- } \\
\text { PNL-Neuquén }\end{array}$ & $\begin{array}{c}\text { madera muerta } \\
\text { de } N \text {. obliqua }\end{array}$ \\
\hline & 4489 & $\begin{array}{l}\text { Pto. Pucará- } \\
\text { PNL-Neuquén }\end{array}$ & $\begin{array}{c}\text { madera muerta } \\
\text { de } N \text {. obliqua }\end{array}$ \\
\hline & 4464 & $\begin{array}{l}\text { Chachín-PNL- } \\
\text { Neuquén }\end{array}$ & $\begin{array}{c}\text { madera muerta } \\
\text { de } N \text {. obliqua }\end{array}$ \\
\hline & 4466 & $\begin{array}{l}\text { Pto. Pucará- } \\
\text { PNL-Neuquén }\end{array}$ & $\begin{array}{c}\text { madera muerta } \\
\text { de } N \text {. obliqua }\end{array}$ \\
\hline & 4470 & $\begin{array}{l}\text { Chachín-PNL- } \\
\text { Neuquén }\end{array}$ & $\begin{array}{c}\text { madera muerta } \\
\text { de } N \text {. obliqua }\end{array}$ \\
\hline
\end{tabular}

Referencia: PNLA: Parque Nacional Los Alerces; PNNH: Parque Nacional Nahuel Huapi; PNL: Parque Nacional Lanín. 
Durante la incubación se registró y midió cada 4 días el diámetro miceliar en dos ejes cartesianos trazados sobre la tapa de la caja. La tasa de crecimiento miceliar $(\mathrm{Kt})$ se estimó con la función de crecimiento lineal $y=k x+c(y=$ distancia, $x=$ tiempo y $c=$ factor constante) y se expresó en milímetros por día (mm/d) (Zervakis et al., 2001). Cuando los micelios cubrieron $1 / 4$ de la superficie de la placa, se los caracterizó morfológicamente registrando la textura, tipo de borde, color y aroma en todos los medios de cultivo, adaptando la metodología propuesta por Nobles (1965) y Stalpers (1978).

\section{Evaluación del crecimiento miceliar en granos}

Esta parte del trabajo involucró dos etapas: a) Selección de las cepas y b) Preparación de inóculo primario. Para la selección de las cepas se evaluó el vigor de acuerdo a la tasa de crecimiento miceliar promedio $(\mathrm{Kt})$ y a la densidad del micelio (Gaitán-Hernández \& Baéz Rodríguez, 2008). Para este último parámetro se establecieron de manera arbitraria tres categorías: densidad baja (presencia de un micelio sumergido, escaso, distribuido de manera heterogénea), densidad regular (presencia de un micelio aéreo, escaso, distribuido de manera homogénea), y densidad alta (presencia de un micelio aéreo, abundante, distribuido de manera homogénea). Se seleccionaron 3 cepas por especie, según 3 clases: (1) de crecimiento acelerado, con una Kt promedio alta (en comparación con el resto de las cepas), y micelio de densidad alta, (2) de crecimiento medio, con una Kt promedio intermedia (en comparación con el resto de las cepas) y micelio de densidad regular y, (3) de crecimiento lento (en comparación con el resto de las cepas), con una Kt promedio baja, y micelio de densidad baja.

La preparación del inóculo primario se realizó con semillas hidratadas durante $2 \mathrm{~h}$ de centeno (Secale cereale L.) y trigo (Triticum aestivum L.), precocidas posteriormente en agua a 100 ${ }^{\circ} \mathrm{C}$ durante 14 y 12 minutos respectivamente. Inmediatamente se filtraron y colocaron $300 \mathrm{~g}$ de cada grano en frascos de vidrio con tapa perforada y tapón de algodón (Stamets \& Chilton, 1983), y se esterilizaron 2 h en autoclave (Albertó, 2008). La humedad de los granos se calculó sobre $100 \mathrm{~g}$ de grano de acuerdo a lo recomendado por Stamets \&
Chilton (1983) y alcanzó un valor del 60\%. Cada frasco se inoculó a temperatura ambiente, con 4 discos de 0,5 cm (Ø) de APD con micelio de cada una de las cepas seleccionadas, y se incubó en oscuridad a $25 \pm 1{ }^{\circ} \mathrm{C}$ durante 30 días. Se registró el crecimiento del micelio cada 3 días en cuatro puntos equidistantes entre sí, ubicados en la parte lateral del frasco, perpendicular al borde superior de los granos. El avance del micelio se midió a partir de cada punto, en dirección a la base del frasco, una vez colonizada la parte superior del mismo. Se calculó el promedio para cada par de datos y se estimó la tasa de crecimiento adaptando la metodología propuesta por Zervakis et al. (2001) para crecimiento en placas. Se describió la distribución (homogénea o heterogénea) y la densidad de micelio (poco denso, denso, muy denso) en los granos de cereal.

\section{Diseño experimental y análisis estadístico}

Para la estimación de la tasa de crecimiento miceliar de las especies cultivadas a diferentes temperaturas, se aplicó un diseño al azar con arreglo factorial de 7 (especies) x 3 (temperaturas de cultivo), con tres réplicas que correspondieron a distintas cepas de cada especie por tratamiento. A su vez, de cada combinación cepa-temperatura se efectuaron 3 réplicas.

Para evaluar la tasa de crecimiento miceliar de las especies cultivadas en diferentes medios de cultivo, se aplicó un diseño al azar con diseño factorial de 7 (especies) x 3 (medios de cultivo), con cinco réplicas que correspondieron a distintas cepas de cada especie por tratamiento. A su vez, de cada combinación cepa-medio se efectuaron 5 réplicas.

Para el crecimiento en granos, se aplicó arreglo factorial de 7 (especies) x 2 (tipos de granos), con tres réplicas por tratamiento que correspondieron a las tres cepas seleccionadas en el ensayo en placas. De cada combinación cepa-grano se efectuaron 3 réplicas.

Para detectar diferencias entre las especies en cada ensayo, se realizaron análisis de varianza de dos vías de clasificación. Para evaluar efecto de la temperatura, las variables clasificatorias fueron las temperaturas y las especies, y la variable respuesta los promedios de las tres réplicas de cada cepa. Para evaluar crecimiento en placas y en granos, las variables clasificatorias 


\section{V. Toledo y C. Barroetaveña - Caracterización miceliar de hongos silvestres comestibles}

fueron los medios y las especies en ambos casos, y las variables de respuesta los promedios de las cinco y las tres réplicas de cada especie respectivamente. Para detectar diferencias en la tasa de crecimiento entre cepas para cada ensayo, se analizó cada especie por separado mediante análisis de varianza de dos vías de clasificación.

En el caso de interacciones significativas, se realizaron contrastes ortogonales entre los factores intervinientes. Los promedios se separaron de acuerdo con la prueba de comparación "a posteriori" aplicando el test de rangos múltiples de Duncan $(\mathrm{p}<0,05)$. Los análisis estadísticos se realizaron con el paquete InfoStat versión 2011 (Di Rienzo et al., 2011).

\section{Resultados y Discusión}

Determinación de la temperatura óptima de crecimiento

La tendencia general muestra una mayor tasa de crecimiento de las especies estudiadas a $25^{\circ} \mathrm{C}$ (Fig. 1), y crecimientos variables y significativamente diferentes entre las cepas de la misma especie crecidas a $25^{\circ} \mathrm{C}$, a excepción de $A$. vitellinus que presentó un crecimiento similar $(\mathrm{p}=0,411)$ entre dos cepas (Fig.1). Las diferentes cepas cultivadas a diferentes temperaturas de incubación para cada especie mostraron diferencias significativas tanto para los factores cepas $(\mathrm{p}<0,0001)$ y temperaturas $(\mathrm{p}<0,0001)$, como para su
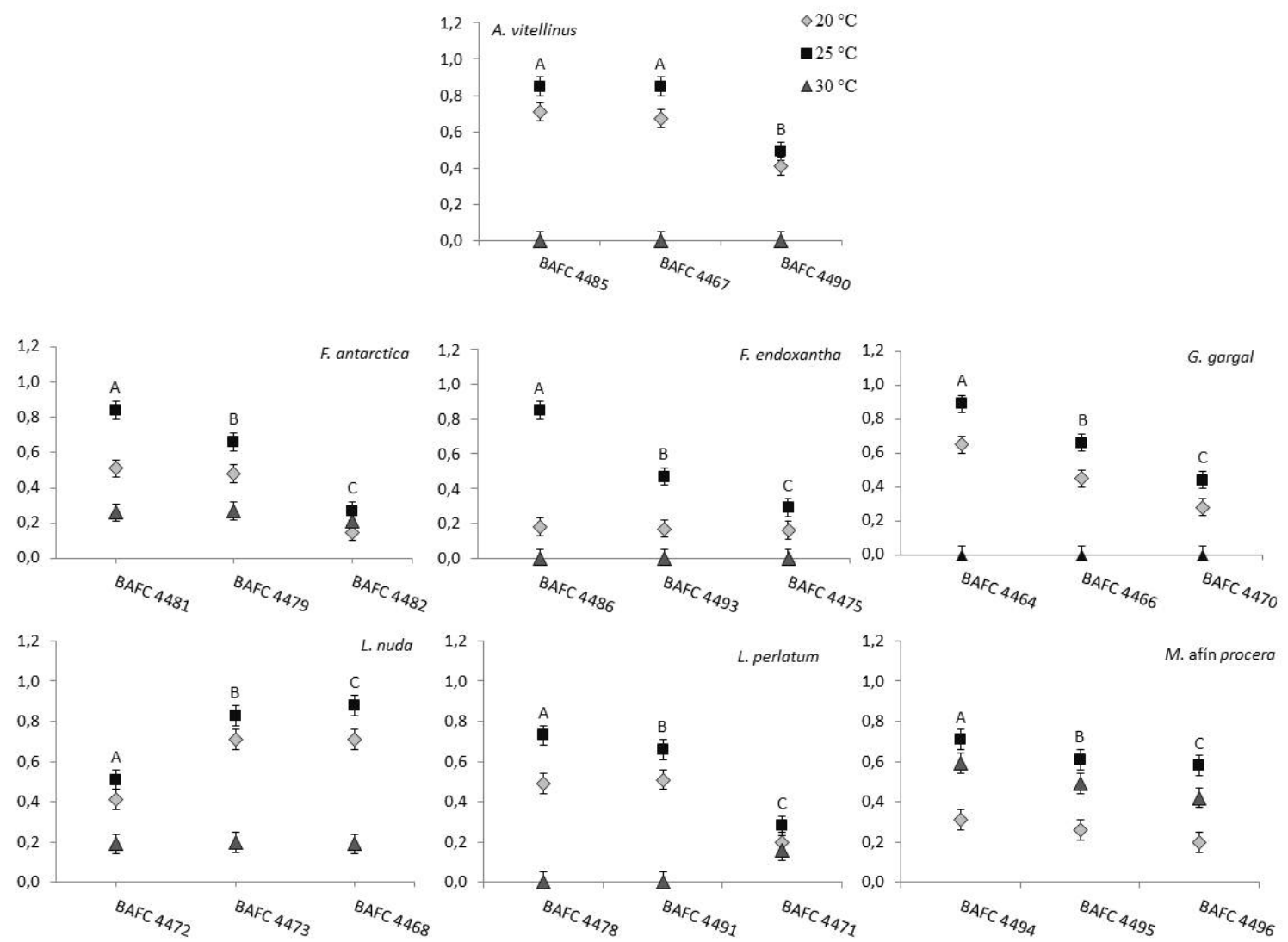

Fig. 1. Tasa de crecimiento miceliar ( $\mathrm{mm} / \mathrm{día}$ ) (indicado en el eje de las ordenadas), de cada una de las cepas de siete especies silvestres comestibles (indicadas en el eje de las abscisas), cultivadas a 3 temperaturas. Los valores son medias de 3 réplicas por cada cepa. Letras diferentes sobre los puntos indican diferencias significativas entre cepas de una misma especie a $25^{\circ} \mathrm{C}(p<0.05)$. 
interacción ( $p<0,0001)$, indicando que las cepas evaluadas crecen de diferente manera en cada una de las temperaturas evaluadas, en consecuencia los factores estudiados no actúan independientemente.

Este trabajo representa el primer aporte en relación a la temperatura óptima de crecimiento para la especie $F$. endoxantha, con un valor de 25 ${ }^{\circ} \mathrm{C}$, y para las especies $A$. vitellinus, $F$. antarctica, L. perlatum, cuyo rango óptimo fue entre 20 y 25 ${ }^{\circ} \mathrm{C}$ (Fig 1). Lepista nuda presentó mejor crecimiento vegetativo entre 20 y $25^{\circ} \mathrm{C}$, coincidiendo con los rangos reportados por otros autores (Wright \& Hayes, 1978; Stott, 1998; Gaitán-Hernández \& Báez Rodríguez, 2008), al igual que Grifola gargal, coincidiendo parcialmente con lo obtenido por Postemsky et al. (2006) y por FIA (2009), quienes indicaron que su crecimiento óptimo ocurrió en un estrecho rango de temperaturas, entre los 18 y $20^{\circ} \mathrm{C}$. Macrolepiota afín procera exhibió una temperatura óptima de crecimiento de 25 a $30{ }^{\circ} \mathrm{C}(\mathrm{p}=0,33)$, semejante a lo hallado por Shim et al. (2005).

Crecimiento miceliar en placas de Petri con diferentes medios de cultivo

La Kt mostró diferencias significativas entre especies $(p<0,05)$ y entre medios $(p<0,0001)$. No se detectaron diferencias significativas para la interacción entre especies y medios a $25 \pm 1{ }^{\circ} \mathrm{C}$ $(\mathrm{p}=0,9)$. El medio de cultivo más rápidamente invadido por las 7 especies fue APD (promedio entre especies $0,86 \mathrm{~mm} /$ día), seguido por $\mathrm{AM}$ (promedio entre especies $0,75 \mathrm{~mm} /$ día) y AEM (promedio entre especies 0,62 mm/día) (Fig. 2). Los resultados coinciden y validan para estas especies reportes que indican que la mayoría de los hongos comestibles responden mejor en APD para iniciar la activación de las cepas y en su multiplicación posterior (Osman et al., 2009). Sin embargo, algunas cepas se comportaron diferente, como la BAFC 4467 de A. vitellinus $(\mathrm{p}=0,24)$, BAFC 4476 de $F$. endoxantha $(\mathrm{p}=$ $0,22)$, BAFC 4492 y BAFC 4478 de L. perlatum $(p=0,06)$, no difiriendo significativamente en los medios AM y APD; en tanto que la cepa BAFC 4468 de $L$. nuda, presentó valores más altos de $\mathrm{Kt}$ en AEM que en AM, de acuerdo a lo registrado mediante la apertura de la interacción (Fig.2).

Aleurodiscus vitellinus y L. nuda registraron las mayores Kt promedio en comparación con las especies restantes para los 3 medios de cultivo, con un valor cercano a $0,80 \mathrm{~mm} /$ día. En otros estudios realizados sobre $L$. nuda a diferentes temperaturas, se observó una Kt cercana a 0,54 $\mathrm{mm} /$ día en agar malta suplementado con polvo de madera de encino (Quercus ilex L.) (GaitánHernández \& Baéz Rodríguez, 2008) 67,5 \% menor a lo registrado en este trabajo $(0,80 \mathrm{~mm} /$ día).

Los resultados de la tasa de crecimiento miceliar de las diferentes cepas de cada especie en APD mostraron diferencias significativas para todas las cepas de las especies A. vitellinus, $F$. endoxantha y L.perlatum, mientras que las cepas de $F$. antarctica, $G$. gargal, $M$. afín procera y $L$. nuda presentaron menor variabilidad (Fig. 2).

Características morfológicas de las diferentes cepas en placas de Petri

La morfología de cada cepa mostró variaciones de acuerdo al medio en el que fueron cultivadas (Tabla 2). Las cepas crecidas en APD se caracterizaron por un micelio con una densidad escasa a abundante con diferentes tipos de borde y textura, en comparación a las cepas cultivadas en AEM y AM, que desarrollaron micelios menos abundantes (Fig. 3). La diferente morfología de crecimiento del micelio de cada una de las especies en los diferentes medios de cultivo se sustenta en que bajo condiciones nutricionales más favorables (representada por APD en este caso), los hongos incrementan la ramificación de sus hifas $\mathrm{y}$, por consiguiente, la cantidad de biomasa, aumentando la eficiencia de suministro de nutrientes al incrementarse su área superficial (Jennings \& Lysek, 1999; Harris, 2008).

En particular, se observaron diferencias morfológicas para las especies G. gargal y L. nuda crecidas en los diferentes medios de cultivo, mientras que $A$. vitellinus, $F$. antarctica, $F$. endoxantha, L. perlatum y $M$. afín procera mostraron similitudes en el tipo de borde, en la textura y en la densidad del micelio en los tres medios (Tabla 2). Grifola gargal cultivada en APD desarrolló un micelio aéreo más abundante que al ser cultivada en AM y AEM, y mostró en AM un aspecto de mata diferente al reportados por Rajchenberg (2002) que lo describió como afelpado-farinoso, coincidiendo en los bordes regulares y sumergidos descriptos en este trabajo. 
C. V. Toledo y C. Barroetaveña - Caracterización miceliar de hongos silvestres comestibles

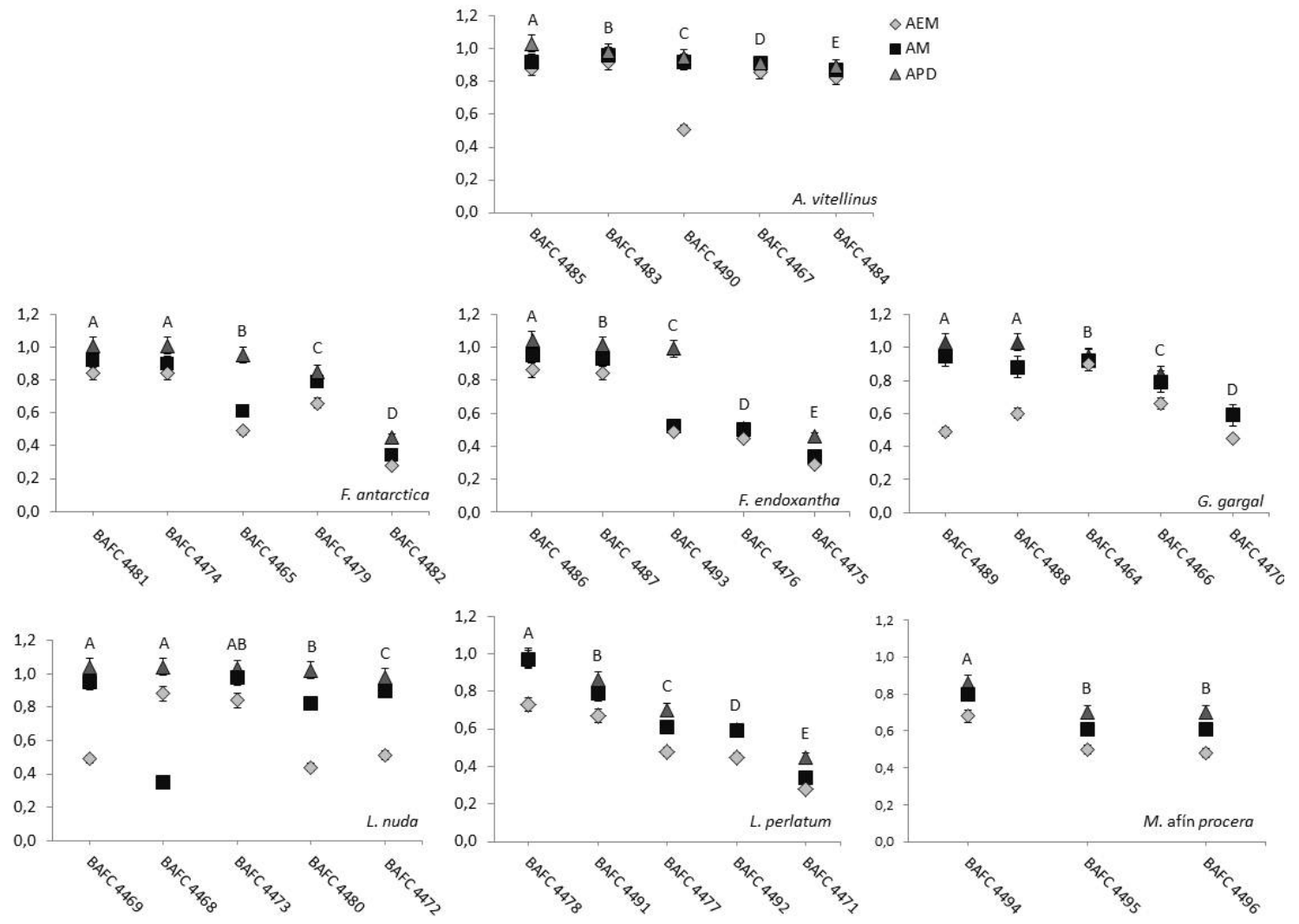

Fig. 2. Tasa de crecimiento miceliar ( $\mathrm{mm} / \mathrm{día}$ ) (indicado en el eje de las ordenadas), de cada una de las cepas (indicadas en el eje de las abscisas) de las especies silvestres comestibles, cultivadas en 3 medios de cultivo: APD: medio de agar con papa y dextrosa; AM: medio de agar con malta y maíz; AEM: medio agar con extracto de malta. Temperatura de medición $25 \pm 1^{\circ} \mathrm{C}$. Los valores son medias de 3 réplicas por cada cepa. Letras diferentes sobre los puntos indican diferencias significativas entre cepas de una misma especie en APD ( $p<0,05$, Duncan).

Tabla 2. Características morfológicas (textura, tipo de borde y densidad) registradas sobre los micelios de las cepas de cada una de las especies sobre tres medios de cultivo.

\begin{tabular}{|c|c|c|c|}
\hline Especies & Textura & Tipo de borde & Densidad \\
\hline A. vitellinus & Afelpada* & Irregulares* & Medianamente abundante* \\
\hline F. antarctica & Sub-afelpada* & Regular/sumergido* & Escaso/heterogéneo* \\
\hline F. endoxantha & Sub-afelpada* & Regular/sumergido* & Escaso/heterogéneo* \\
\hline G. gargal & Algodonosa* & Regular/sumergido* & Abundante* \\
\hline \multirow{3}{*}{ L. nuda } & Algodonosa zonada" & Ondulado/aéreo" & Escaso\# \\
\hline & Algodonosa zonada/afelpada* & Irregular & Medianamente abundante ${ }^{*}$ \\
\hline & Afelpada ${ }^{\#}$ & Regular/sumergido\# & Poco abundante \\
\hline L. perlatum & Afelpada* & Regulares* & Escaso/homogéneo* \\
\hline M. afín procera & Afelpada ramificada* & Irregulares/aéreo* & Escaso/homogéneo* \\
\hline
\end{tabular}

Referencia: "Agar de papa dextrosado; "Agar con extracto de malta y Agar maíz. 


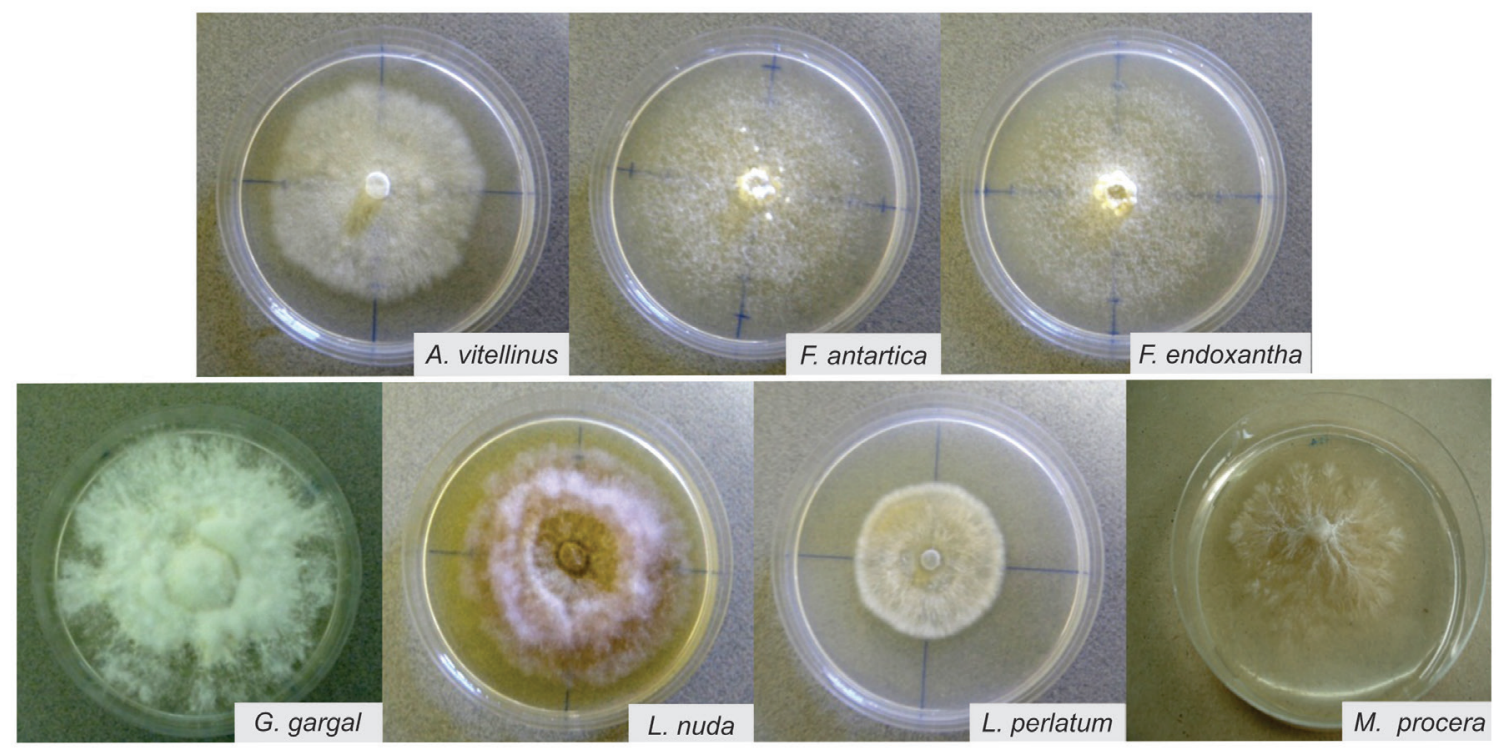

Fig. 3. Morfología de siete especies silvestres comestibles cultivadas en APD (medio de agar con papa y dextrosa) a $25 \pm 1^{\circ} \mathrm{C}$.

Lepista nuda presentó mejores características de vigor en APD, con una textura algodonoso/zonada y algodonoso/afelpada en algunas de las cepas, y una densidad del micelio abundante y medianamente abundante en comparación con las cepas crecidas en AEM y AM. Sin embargo, otros autores han observado un micelio lineal a algodonoso en $L$. nuda cultivada en agar malta (Stamets \& Chilton, 1983).

El micelio de A. vitellinus se caracterizó por una textura afelpada y una densidad medianamente abundante en los tres medios de cultivo, pero se observó la formación de anillos concéntricos creciendo en AEM. El color del micelio se mantuvo constante en los tres medios de cultivo para todas las cepas estudiadas, siendo el blanco el color predominante, a excepción de $F$. antarctica que presentó sectores de color ocre y de $L$. nuda que se caracterizó por poseer tonos violáceos, similar a lo descripto por Stamets (1993) y Stamets \& Chilton (1983).

El aroma fue variable entre las especies, térreo en $L$. nuda, a vegetales en L. perlatum y almendrado en G. gargal, tal como reporta Rajchenberg (2002) para esta última. Trabajos anteriores señalaron un aroma ligeramente frutado para $F$. antarctica (Rajchenberg \& Greslebin, 1995), lo cual no fue detectado en este trabajo. Para las especies restantes no se detectó aroma particular.
Ninguna de las especies estudiadas equiparó en velocidad de crecimiento a la cepa comercial de $P$. ostreatus $(2,18 \mathrm{~mm} /$ día); los valores de $\mathrm{Kt}$ de $A$. vitellinus y $L$. nuda fueron $40 \%$ más bajos que para P. ostreatus. Tampoco equipararon los valores de $\mathrm{Kt}$ de otras especies cultivadas como $P$. pulmonarius (Fr.) Quél. (4,5 mm/día) o L. edodes (5,55 mm/día), según lo reportado por Suárez \& Holguín (2011). Si bien las especies patagónicas no pueden competir en crecimiento con otras especies ampliamente cultivadas, poseen atributos tales como la textura carnosa, aroma y colores llamativos, además de su rareza en el mercado, que las tornan atractivas para su cultivo y comercialización. Otros estudios realizados sobre cepas de hongos silvestres comestibles de alta montaña de México (AranaGabriel et al., 2014), encontraron velocidades de crecimiento aun menores que las registradas para las especies estudiadas en este trabajo: Psathyrella spadicea (P. Kumm.) Singer con 0,35 mm/día y Floccularia luteovirens (Alb. y Schwein.) Pouzar y Clitocybe squamulosa (Pers.) P. Kumm con 0,38 $\mathrm{mm} /$ día.

\section{Crecimiento miceliar en granos}

La producción de inóculo primario no mostró diferencias significativas entre los granos de 


\section{V. Toledo y C. Barroetaveña - Caracterización miceliar de hongos silvestres comestibles}

trigo y centeno para el total de especies evaluadas (Tabla 3), por lo que resulta indistinto el uso de cualquiera de ambos, debiendo privilegiarse la disponibilidad, el costo y la calidad del grano disponible en la elección. El trigo ha sido utilizado con buenos resultados en Agrocybe cylindracea (DC.: Fr.) Maire (Stamets, 1993) y también en Pleurotus ostreatus y P. pulmonarius (Martínez-Carrera et al., 1985, 1988). El centeno ha sido recomendado para la producción de semilla de A. bisporus y L. edodes (Stamets \& Chilton, 1983).

La Kt fue significativamente diferente entre cepas $(\mathrm{p}<0,0001)$ para todas las especies, y entre los granos $(\mathrm{p}<0,05)$ para $A$. vitellinus, F. endoxantha, G. gargal, L. nuda, L. perlatum y $M$. afín procera (Tabla 3). En tanto, el efecto interacción fue significativo en G. gargal (p $=0,01)$, L. perlatum y $M$. afín procera $(\mathrm{p}<$ $0,0001)$, indicando que las cepas crecen de diferente manera en cada uno de los granos ensayados.

En relación a la morfología, todas las cepas de L. nuda, A. vitellinus y G. gargal desarrollaron un micelio abundante, aéreo, de textura algodonosa distribuido de manera heterogénea sobre los granos, a diferencia de las cepas de F. antarctica, $F$. endoxantha, L. perlatum y $M$. afín procera que mostraron un micelio de desarrollo incipiente. En general la tasa de crecimiento miceliar promedio observada sobre ambos tipos de granos, mostró variabilidad entre las cepas evaluadas, en tanto que la morfología no varió entre cepas.

Las $\mathrm{Kt}$ de todas las cepas crecidas sobre ambos granos fueron significativamente menores a la cepa comercial de $P$. ostreatus $(\mathrm{p}<0,05)$, cuyo valor fue de $5,92 \mathrm{~mm} /$ día en trigo y 4,96 $\mathrm{mm} /$ día en centeno. Asimismo, la colonización total de los granos por parte de la cepa comercial de $P$. ostreatus se produjo a los 18 días, y se caracterizó por el desarrollo de un micelio blanco, muy denso, distribuido de manera homogénea en ambos granos. Las cepas de las especies investigadas, en cambio, solamente cubrieron alrededor de un $50 \%$ de la totalidad de los granos al cabo de los 30 días de evaluación.

Si bien la velocidad de crecimiento miceliar constituye un parámetro fundamental en la selección de cepas, medios y sustratos, dado que contribuye a evitar el arribo de agentes

Tabla 3. Tasa de crecimiento miceliar promedio ( $\mathrm{mm} / \mathrm{día})$ de las diferentes cepas y entre los diferentes granos para siete especies de hongos comestibles \pm (D.E.).

\begin{tabular}{|c|c|c|c|c|c|}
\hline Especies & & Cepas & & Trigo & Centeno \\
\hline A. vitellinus & $\begin{array}{c}4485 \\
* 1,73 \pm(0,05)^{\mathrm{A}}\end{array}$ & $\begin{array}{c}4490 \\
1,45 \pm(0,14)^{B}\end{array}$ & $\begin{array}{c}4467 \\
1,43 \pm(0,12)^{\mathrm{C}}\end{array}$ & $* * 1,54 \pm(0,22)^{A}$ & $1,53 \pm(0,32)^{B}$ \\
\hline F. antarctica & $\begin{array}{c}4481 \\
* 1,44 \pm(0,12)^{A}\end{array}$ & $\begin{array}{c}4479 \\
1,42 \pm(0,32)^{B}\end{array}$ & $\begin{array}{c}4482 \\
1,38 \pm(0,02)^{c}\end{array}$ & $1,41 \pm(0,12)^{\mathrm{A}}$ & $1,41 \pm(0,02)^{A}$ \\
\hline F. endoxantha & $\begin{array}{c}4486 \\
* 1,43 \pm(0,21)^{A}\end{array}$ & $\begin{array}{c}4493 \\
1,41 \pm(0,03)^{A}\end{array}$ & $\begin{array}{c}4475 \\
1,37 \pm(0,05)^{\mathrm{B}}\end{array}$ & $* 1,40 \pm(0,01)^{A}$ & $1,41 \pm(0,02)^{B}$ \\
\hline G. gargal & $\begin{array}{c}4464 \\
* 1,60 \pm(0,03)^{A}\end{array}$ & $\begin{array}{c}4466 \\
1,45 \pm(0,03)^{B}\end{array}$ & $\begin{array}{c}4470 \\
1,41 \pm(0,26)^{C}\end{array}$ & $* 1,49 \pm(0,01)^{A}$ & $1,48 \pm(0,04)^{B}$ \\
\hline L. nuda & $\begin{array}{c}4473 \\
* 1,97 \pm(0,02)^{A}\end{array}$ & $\begin{array}{c}4472 \\
1,47 \pm(0,02)^{B}\end{array}$ & $\begin{array}{c}4468 \\
1,39 \pm(0,02)^{c}\end{array}$ & $* * 1,62 \pm(0,21)^{A}$ & $1,60 \pm(0,13)^{B}$ \\
\hline L. perlatum & $\begin{array}{c}4478 \\
* 1,38 \pm(0,01)^{A}\end{array}$ & $\begin{array}{c}4491 \\
1,30 \pm(0,22)^{B}\end{array}$ & $\begin{array}{c}4471 \\
1,26 \pm(0,22)^{B}\end{array}$ & $* 1,36 \pm(0,31)^{A}$ & $1,26 \pm(0,23)^{\mathrm{B}}$ \\
\hline $\begin{array}{l}\text { M. afín } \\
\text { procera }\end{array}$ & $\begin{array}{c}4494 \\
* 1,28 \pm(0,03)^{A}\end{array}$ & $\begin{array}{c}4495 \\
1,36 \pm(0,04)^{B}\end{array}$ & $\begin{array}{c}4496 \\
1,26 \pm(0,25)^{C}\end{array}$ & $* 1,35 \pm(0,01)^{A}$ & ${ }^{*} 1,25 \pm(0,04)^{\mathrm{B}}$ \\
\hline
\end{tabular}

Referencia: ${ }^{*} p<0,0001 ;{ }^{* *} p<0,05$. Valores promedios seguidos por letras diferentes en cada fila indican diferencias significativas para cada efecto ( $p<0,05$, Duncan). 
contaminantes, no representa necesariamente una ventaja en el desarrollo de los basidiomas (Stamets, 1993; Salmones et al., 1997). Por ello, serán necesarias futuras investigaciones que permitan encontrar aquellos sustratos orgánicos sobre los cuales las especies aquí estudiadas puedan ser domesticadas de una manera óptima. Probablemente L. nuda, G. gargal y $M$. afín procera, de las que existen reportes sobre producción en sustrato (Shim et al., 2005; Castro et al., 2014, Harada et al., 2015) serán más recomendables para iniciar cultivos experimentales. Estas tres especies, junto a $A$. vitellinus, que mostró buen crecimiento miceliar tanto en placas como en granos, presentan características organolépticas atractivas para su cultivo intensivo. La domesticación de estas especies de hongos del bosque nativo Patagónico permitiría generar de manera continua un producto alimenticio interesante y novedoso, y eventualmente podría contribuir a la conservación de algunas de estas especies silvestres asociadas a estas masas boscosas, en caso de que se intensifique su aprovechamiento (eg. conservación ex situ).

\section{Conclusiones}

La mayor tasa de crecimiento miceliar en placas de Petri y en granos, con micelios de densidad entre abundante y medianamente abundante en relación al resto de las especies estudiadas, indican que A. vitellinus y L. nuda serían las más promisorias para ser utilizadas en ensayos para su cultivo intensivo.

Las cepas de $A$. vitellinus, $F$. endoxantha y L. perlatum mostraron mayor variabilidad en la tasa de crecimiento en distintos tipos de medios de cultivo en comparación a las cepas de $F$. antarctica, G. gargal y $M$. afín procera.

La temperatura óptima de crecimiento para todas las especies fue $25^{\circ} \mathrm{C}$, y el medio de cultivo con mejores crecimientos fue PDA.

Si bien las especies con mejores crecimientos reportaron valores $40 \%$ más bajos que la cepa comercial de $P$. ostreatus, todas poseen atributos organolépticos particulares, y serían novedosas para un mercado gourmet, con una fuerte impronta de origen.

\section{Agradecimientos}

Este trabajo fue financiado por los proyectos PICT-2011-0118 FONCyT (Argentina) y UNPSJBFi $\mathrm{N}^{\circ} 001$ de la Universidad Nacional de la Patagonia San Juan Bosco.

\section{Bibliografía}

ALBERTÓ, E. 2008. Cultivo intensivo de hongos comestibles. Como cultivar champiñones, Gírgolas, Shitake y otras especies. Ed. Hemisferio Sur, Buenos Aires.

ARANA-GABRIEL, Y., C. BURROLA-AGUILAR, R. GARIBAY-ORIJEL \& S. FRANCO-MAASS. 2014.

Obtención de cepas y producción de inóculo de cinco especies de hongos silvestres comestibles de alta montaña en el centro de México. Rev. Chapingo Ser. Cie. 20: 213-226.

ARORA, D. 1986. Mushrooms demystified, 2da edición. Ten Speed Press, Berkeley, CA.

BOA, E. 2004. Wild edible fungi. A global overview of their use and importance to people. Non-wood forest products. Food \& Agriculture Organization of the United Nations, Volumen: 17, Roma.

BRAN, M., O. MORALES, R. FLORES, R. CÁCERES \& N. GURRIARÁN. 2009. Cultivo de cepas guatemaltecas del hongo comestible Tx'yol B'aqman (Agrocybe cylindracea (DC.) Maire): caracterización y producción de cuerpos fructíferos. Informe Técnico Final. Dirección General de Investigación. USAC.

CASTRO, F., A. MORENO, A. GARCÍA \& F. ORTIZ. 2014. El cultivo de Lepista nuda en sustrato con hojas de olivo para el aprovechamiento de subproductos agroindustriales en almazaras. Anales Biol. 36: 1117.

CHANG, S. T. \& P. G. MILES. 2004. Mushrooms: Cultivation, Nutritional Value, Medicinal Effect, and Environmental Impact. CRS Press, Florida.

DI RIENZO, J. A., F. CASANOVES, M. G. BALZARINI, L. GONZALEZ, M. TABLADA \& C.W. ROBLEDO. 2011. Grupo InfoStat, FCA, Universidad Nacional de Córdoba, Córdoba.

FIA. 2009. Resultados y Lecciones en el Cultivo del Hongo Gargal. Fundación para la Innovación Agraria. Serie Experiencias de Innovación para el Emprendimiento Agrario $\mathrm{N}^{\circ} 30$. VIII, Región del BioBío.

FURCI, G. 2007. Fungi Austral. Guía de campo de los hongos más vistosos de Chile. Andros impresores, Concepción.

GAITÁN-HERNÁNDEZ, R.\& I. BAÉZ RODRIGUEZ. 2008. Crecimiento miceliar de cepas silvestres nativas de Lepista nuda, en medios de cultivo con 


\section{V. Toledo y C. Barroetaveña - Caracterización miceliar de hongos silvestres comestibles}

diferentes suplementos orgánicos. Rev. Mex. Mic. 26: 41-49.

GAMUNDÍ, I. J. \& E. HORAK. 1993. Hongos de los bosques andino-patagónicos. Vázquez Mazzini, Buenos Aires.

GORJÓN, S., A. G. GRESLEBIN \& M. RAJCHENBERG. 2013.The genus Aleurodiscus sl (Stereaceae, Russulales) in the Patagonian Andes. Mycol. Prog. 12: 91-108.

GUINBERTEAU, J. \& J. M. OLIVIER. 1991. Improvement of Lepista species cultivation, technical factors and selection of strains. In: MAHER, M. J. \& A. A. BALKEMA (Eds.). Science and cultivation of edible fungi, pp.615621. A. A. Balkema Publishers, Brookfield.

HARADA, E., T. MORIZONO, T. SUMIYA \& S. MEGURO. 2015. Production of AndeanPatagonic edible mushroom Grifola gargal on wood-based substrates. Mycoscience 56: 616-621.

HARRIS, S. D. 2008. Branching of fungal hyphae: Regulation, mechanisms and comparison with other branching systems. Mycologia 100: 823832.

JENNINGS, D. H. \& G. LYSEK. 1999. Fungal Biology. Ed. Springer, New York.

LEÓN-AVENDAÑO, H., R. MARTÍNEZ-GARCÍA, P. CABALLERO GUTIÉRREZ \& D. MARTÍNEZCARRERA. 2013. Caracterización de dos cepas de Pleurotus djamor nativas de Oaxaca, México. Rev. Mex. Cienc. Agric. 6:1285-1291.

MARTÍNEZ-CARRERA, D., C. SOTO \& G. GUZMÁN. 1985. Cultivo de Pleurotus ostreatus en pulpa de café con paja como substrato. Rev. Mex. Mic. 1:101-108.

MARTÍNEZ-CARRERA，D., P. MORALES \& M. SOBAL. 1988. Cultivo de Diversas cepas Mexicanas de Pleurotus ostreatus sobre pulpa de café y paja de cebada. Rev. Mex. Mic. 4:153-160.

MOSER, M.H. 1978. Keys to Agarics and Boleti (Polyporales Boletales Agaricales Russulales). Roger Phillips Publ. UK.

NOBLES, M. 1965. Identification of cultures of woodinhabiting Hymenomycetes. Can. J. Bot. 43: 1097-1139.

OSMAN, M., F. HASSAN, O. KHATTAB, W. AHMED \& H. EL-HENAWY. 2009. Physiological studies on growth of two differ strains of Lentinus edodes. Aust. J. Basic Appl. Sci. 3: 4094-4103.

PHILLIPS, R. 1981. Mushrooms and other fungi of Great Britain and Europe. Pan Books, London.

POSTEMSKY, P., R. GONZÁlEZ MATUTE, D. FIGLAS \& N. CURVETTO. 2006. Optimizing Grifola sordulenta and Grifola gargal growth in agar and liquid nutrient media. Micol. Aplic. Int. 18: 7-12.
RAJCHENBERG, M. 2002. The Genus Grifola (Aphillophorales, Basidiomycota) in Argentina revisisted. Bol. Soc. Argent. Bot., 37: 19-27.

RAJCHENBERG, M. 2006. Los Poliporos (Basidiomycetes) de los Bosques Andino Patagónicos de Argentina. Primera edición. Editorial Borntraeger, Stuttgart.

RAJCHENBERG, M., GRESLEBIN, A. 1995. Cultural characters, compatibility tests and taxonomic remarks of selected polypores of the Patagonian Andes forests of Argentina. Mycotaxon, 56: 325 346.

SALMONES, D., R. GAITÁN-HERNÁNDEZ, R. PÉREZ \& G. GUZMÁN. 1997. Estudios sobre el género Pleurotus. VIII. Interacción entre crecimiento micelial y productividad. Rev. Iberoam. Micol., 14: 173-176.

SHIM, S.M., Y.H.OH, K.R. LEE, S.H. KIM, K.H. IM, J.W. KIM, U.Y. LEE, J.O. SHIM, M.J. SHIM, M.W. LEE, H.S. RO. H.S. LEE \& T.S. LEE. 2005 The characteristics of cultural conditions for the mycelial growth of Macrolepiota procera. Mycobiology, 33: 15-18.

STALPERS, J.A. 1978. Identification of woodinhabiting Aphyllophorales in pure culture. Stud. Mycol. 16: 1-248.

STAMETS, P. 1993. Growing Gourmet and Medicinal Mushrooms. Third edition. Berkeley: Ten Speed Press, Berkeley, Ca.

STAMETS, P. \& J.S. CHILTON. 1983. The mushroom cultivator. A practical guide to growing mushrooms at home. Washington: Agarikon Press, Olympia, Wa.

STOTT, K. 1998. Characteristics of Australian edible fungi in the genus Lepista and investigation into factors affecting cultivation. Tesis doctoral. University of Western Sydney Hawkesbury, Sydney.

SUÁREZ, C. \& M.S. HOLGUÍN. 2011. Evaluación de medios de cultivo sintéticos y cereales para la producción de semilla de setas comestibles. Rev. Colomb. Cienc. Hortic. 5: 130-140.

TOLEDO, C.V., C. BARROETAVEÑA \& M. RAJCHENBERG. 2014. Fenología y variables ambientales asociadas a la fructificación de hongos silvestres comestibles de los bosques andino-patagónicos en Argentina. Rev. Mex. Mic. 85: 1093-1103.

TOLEDO, C., C. BARROETAVEÑA \& M. RAJCHENBERG. 2016. Hongos comestibles silvestres de la región Andino Patagónica de la Argentina. Manual No 16. Esquel: Centro Forestal CIEFAP.

TOLEDO, C. 2016. Hongos comestibles silvestres de los bosques nativos de Nothofagus spp. en el norte y centro de la Patagonia andina de la 
Bol. Soc. Argent. Bot. 52 (3) 2017

Argentina., 194p. Tesis (Doctorado) - Universidad del Comahue, Facultad de Ciencias Naturales. S.C. Bariloche.

VALENZUELA-FLORES, E. 2003. Hongos comestibles silvestres colectados en la X región de Chile. Bol. Micol. 18: 1-14.

WANG, Y.C. 1987. Mycology in ancient China. Mycologist 1: 59-61.

WRIGHT, S.H. \& W.A. HAYES. 1978. Nutrition and fruitbody formation of Lepista nuda (Bull. Ex. Fr.) Cook. In: DELMAS, J.(Ed.). pp. 873-84. Mushroom Science X (part 1). Burdeos.
ZERVAKIS, G., PHILIPPOUSSIS, A., IOANNIDOU, S. \& P. DIAMANTOPOULOU. 2001. Mycelium growth kinetics and optimal temperature conditions for the cultivation of edible mushroom species on lignocellulosic substrates. Folia microbial. 46: 231-234.

Recibido el 16 de diciembre de 2016, aceptado el 26 de junio de 2017. 